\title{
A Systematic Assessment of Service Delivery in Preliminary Care Unit at a State Hospital in Sri Lanka
}

\author{
S.M.N.S. Maleesha MALLAWARACHCHI ${ }^{1 *}$
}

Mallawarachchi, S.M.N.S.M. (2021). A systematic assessment of service delivery in Preliminary care unit at a state hospital in Sri Lanka. Journal of Health Systems and Policies (JHESP), 3,41-54.

\section{ABSTRACT}

Preliminary Care Unit is the Sri Lankan model of the emergency department in the state health facilities above the level of Base Hospitals in the country. Provision of prompt, quality, and comprehensive patient care without any unnecessary delay, minimizing unnecessary admissions to wards, and reducing the cost of patient management were the objectives of establishing such units. The current study aimed to systematically assess the process of service delivery in the Preliminary Care Unit of District General Hospital, Gampaha. The study was descriptive with both cross-sectional and retrospective components and was conducted in 2019 . Study instruments and techniques included are key informant interviews, focus group discussions, the desk review of secondary data, and direct observation. Key informant interviews with the medical officer-in-charge and nursing sister in charge of the unit were

\footnotetext{
${ }^{1}$ Ministry of Health, Colombo, Sri Lanka

*Corresponding author: S.M.N.S.M. MALLAWARACHCHI, navodasandamali79@gmail.com
} 
conducted by the principal investigator. Two focus group discussions were conducted, with ten randomly selected medical officers and 8 nursing officers with a minimum one-year of work experience at the Preliminary Care Unit. Data gathered from the desk review of the secondary data from the admission book, Bed Head Tickets, and record of monthly statistics of the preliminary care unit using a checklist to ascertain the total number of admissions and their outcome in 2018. All admissions of a randomly selected week of 2018 were studied to assess the age and gender distribution and they were classified into two categories as medical and surgical, according to the admitting doctor's notes. Direct observation was used to triangulate the data. The major deficiencies of the process of service delivery in the Preliminary Care Unit elicited at the key informant interviews and focus group discussions were overcrowding and excess waiting which were multifactorial in causation. Less organized process flow and inadequate resource availability led to overcrowding and excess waiting.

Keywords: Preliminary Care Unit, Emergency Care, Overcrowding, Waiting, Service Delivery

\section{INTRODUCTION}

In Sri Lanka, therapeutic healthcare is delivered primarily by Government Hospitals which are graded from highest to lowest in rank as Teaching Hospitals, Provincial General Hospitals, District General Hospitals, Base Hospitals, Divisional Hospitals, and Primary Medical Care Units (Sri Lanka Ministry of Health, 2020). In institutions above the level of Base Hospitals, Preliminary Care Units (PCUs) were established to deliver better patient care and improve the service quality of the health institutions (Sri Lanka Ministry of Health, 2012). As per the General circular 01-02/2012 (Sri Lanka Ministry of Health, 2012) aims of establishing PCU in health institutions included, providing prompt, quality, and comprehensive patient care without any unnecessary delay, minimizing unnecessary admissions to wards, and reducing the cost of patient management. 
The PCU could be described as the Sri Lankan model of the emergency department in health facilities. An emergency treatment unit with facilities of resuscitation is an essential component of the PCU (Sri Lanka Ministry of Health, 2012). The establishment of Accident Service at the National Hospital of Sri Lanka in 1991 was recognized as an important landmark in emergency trauma care while Provincial General Hospital Kurunegala pioneered the establishment of an Accident and Emergency Unit in Sri Lanka in 2002 (Sri Lanka Ministry of Health, 2016). Yet, PCUs are the type of emergency department commonly available in state hospitals.

The District General Hospital (DGH), Gampaha is one of the busiest hospitals in Gampaha district, Sri Lanka. Being a DGH, it provides health services in all four main specialties (medicine, paediatrics, surgery, and obstetrics/gynaecology) together with several subspecialties (Sri Lanka Ministry of Health, 2020).

The DGH serves not only people of Gampaha but also those from the other districts as Sri Lanka has no gatekeeping mechanism to legally restrict a hospital's client drainage area (World Health Organization, 2017). The bed capacity is 781 and the average number of patient visits at out-patients department exceeds 300,000 per year (Gampaha District General Hospital Statistics Bulletin, 2016).

The study aimed to assess the service delivery of the PCU in DGH Gampaha in the view of identifying the major deficiencies in the work process and thereby recognizing the necessary interventions to remedy such.

\section{METHODOLOGY}

The study was descriptive with both cross-sectional and retrospective components and was conducted in 2019 at PCU, DGH, Gampaha. 
Study instruments and techniques included, key informant interviews, focus group discussions, the desk review of secondary data, and direct observation. All study instruments were pretested by the pilot study conducted at Base Hospital Dambadeniya in the same district.

Key informant interviews with the medical officer-in-charge and nursing sister incharge of the PCU were conducted by the principal investigator. Two focus group discussions were conducted, with 10 medical officers and 8 nursing officers respectively, who were selected randomly, out of the officers with a minimum one year work of experience at the PCU. All discussions were facilitated by the principal investigator and they were held in both English and their native language (Sinhalese) according to the participants' preference. The key informant interview, guides and formats for focus group discussions were designed to gather data on weaknesses in the process that could inhibit the fulfillment of the said objectives of PCU (Sri Lanka Ministry of Health, 2012) and suggestions for improvement. The deficiencies pointed out by the participants were prioritized and those categorized as major issues were selected for further discussions and analysis. The responses of key informants and participants of focus group discussions were coded, and thematic analysis was carried out.

Data gathered from a desk review of secondary data from the admission book of PCU, Bed Head Tickets, and record of monthly statistics of PCU for the period 01.01.2018 to 31.12.2018 with the use of a checklist. The desk review aimed to ascertain the total number of admissions and the outcome of those, within the aforesaid duration. All admissions in a randomly selected week in 2018 ( $2^{\text {nd }}$ week in the month of October) were studied to assess the age and gender distribution. Randomization of selecting the week was partly to avoid any possible confounding factors that would affect public health- seeking behavior associated with local events/festivals etc. and also to ensure generalizability of results. The total admissions during that week were broadly classified into two categories as medical and surgical, according 
to the admitting doctor's notes. The age of the patients in years was grouped into three categories $0-14,15-64$, and 65 and above, to grasp, paediatric, working and elderly/retired populations respectively (Central Intelligence Agency, 2020).

Direct observations of the process of service delivery of PCU were made by investigators at randomly selected time slots with the objective of triangulation of data gathered from key informants and focus group discussions. The observation was conducted for random14 days from 8 am to $8 \mathrm{pm}$.

Ethical clearance was obtained from the Ethics Review Committee of Medical Research Institute, Sri Lanka (Project number 17/2018). Before the commencement of the study, the principal investigator personally met all the key informants and participants of focus group discussions to explain the aims and methods of the study. All queries from the participants regarding the study were answered by the principal investigator both before and during the study. Due administrative approval was obtained from relevant authorities.

The data were analyzed with SPSS 16.

\section{RESULTS}

The 20 bedded PCU was staffed with 31 doctors and 22 nurses. There were dedicated areas within the premises for resuscitation and minor surgical procedures.

The process of PCU was mapped (Figure 1). 


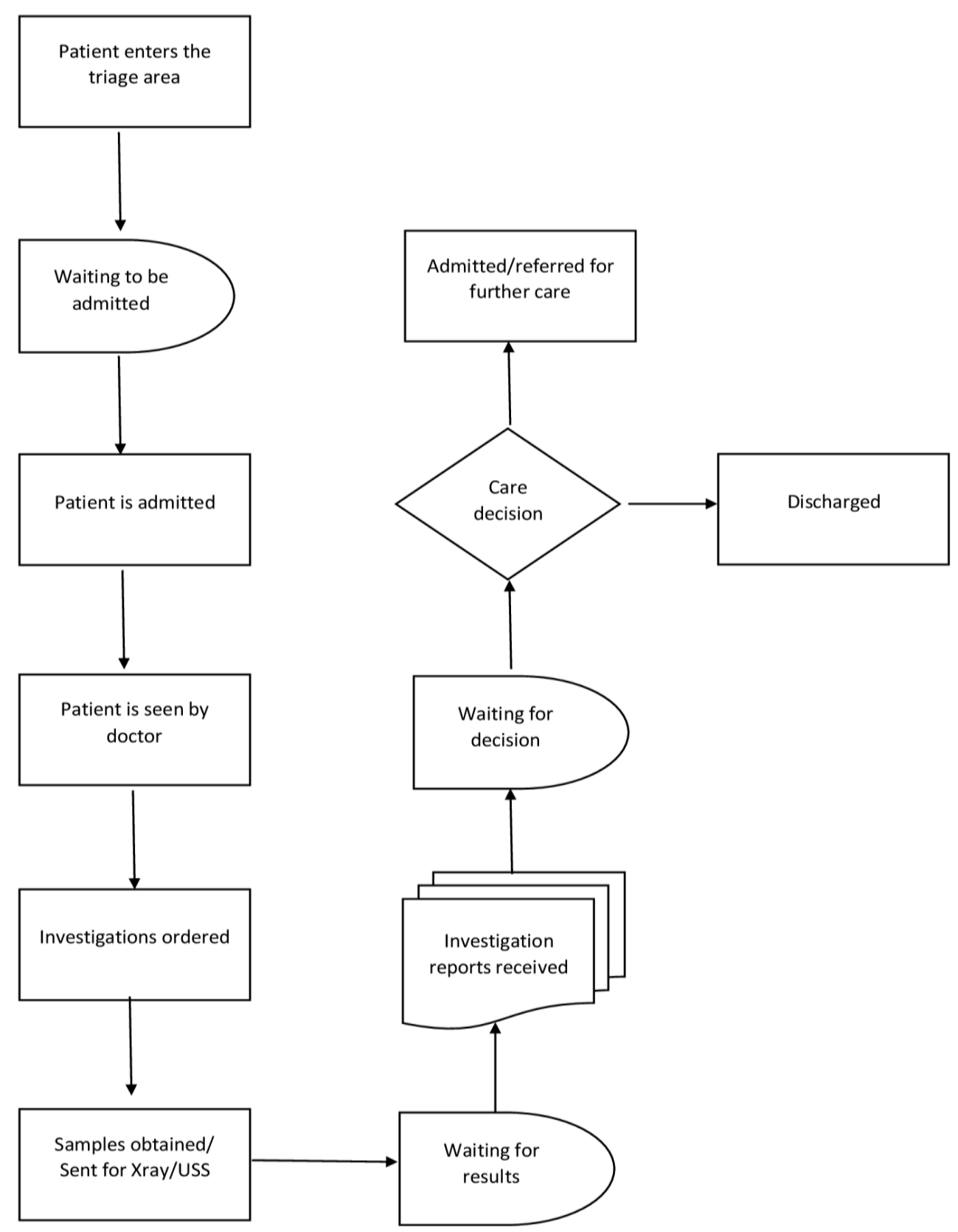

Figure 1: Process of service delivery in Preliminary care unit, DGH Gampaha

A doctor evaluated all the patients admitted to PCU and started treatment. The minor cases were discharged after treatment with necessary medical instructions after a period of observation. Relevant units were informed regarding the critically ill and those who needed admission to wards. The authority to admit the patients to a ward was held by the consultant or the designee of the relevant ward. An intern medical officer was delegated to the PCU by the 
consultants of the respective unit on the days in which the given unit was accepting new admissions. In a case of an emergency, the medical officer PCU could summon all the interns on call if necessary.

Within the PCU to the inward transfer process, there were three phases identified; namely, pre-transfer phase involving PCU care coordination and transfer decision-making, transfer phase involving handoff, and post-transfer phase involving inward care planning and management.

Total admissions to PCU in 2018 was 72182 . Among them $38.96 \%$ were discharged, $52.61 \%$ were admitted to wards and $0.26 \%$ were transferred, $8.06 \%$ were missing or left against medical advice and $0.11 \%$ died.

It was noted that most of the admitted were males (65.08\%) and the majority $(64.06 \%)$ belonged to the 15-64 years age category. Most patients were having medical complaints $(60.32 \%)$ (Table 1).

Table 1: Age, gender, and category of admissions to Preliminary Care Unit, District General Hospital, Gampaha

\begin{tabular}{|c|c|c|}
\hline \multirow{2}{*}{ Attribute } & Category & Percentage \\
\hline Age group (Years) & $0-14$ & 10.94 \\
\cline { 2 - 3 } & $15-64$ & 64.06 \\
\cline { 2 - 3 } & 65 and above & 25 \\
\hline Gender & Male & 65.08 \\
\cline { 2 - 3 } & Female & 34.92 \\
\hline Category of admission & Surgical & 39.68 \\
\cline { 2 - 3 } & Medical & 60.32 \\
\hline
\end{tabular}

*The data depicted in the table were collected from the randomly selected week

The major deficiencies of the process of service delivery in the PCU elicited at the key informant interviews and focus group discussions were overcrowding and excess waiting. The 
top five reasons for overcrowding in PCU according to the responses of the participants, listed according to the importance were,

- Inadequate inpatient capacity

- Lack of priority channel for laboratory tests

- Delays in referral to a judicial medical officer

- Insufficient nursing staff

- Repeated and deliberate insisted self-admissions

The components of the waiting in care process were obtained through key informant interviews and focus group discussions together with the interventions for improvement proposed by the participants (Table 2).

Table 2: The components of waiting in the care process of Preliminary Care Unit, DGH Gampaha, and interventions proposed

\begin{tabular}{|c|c|}
\hline Observed component of waiting & Proposed intervention \\
\hline Prolonged waiting for investigation reports & $\begin{array}{c}\text { Priority channel for investigations sent from } \\
\text { PCU }\end{array}$ \\
\hline Delays in consultants' opinion & $\begin{array}{c}\text { Online opinion seeking and information } \\
\text { sharing facility }\end{array}$ \\
\hline Delays in taking over to wards & $\begin{array}{c}\text { Systematic planning of human resources } \\
\text { especially the minor staff to transfer the } \\
\text { patients timely to wards }\end{array}$ \\
\hline Delays in Judicial medical procedures & $\begin{array}{c}\text { Arranging facilities to keep patients until } \\
\text { they are seen by a judicial medical officer in } \\
\text { a post-acute section adjacent to PCU }\end{array}$ \\
\hline
\end{tabular}

The major deficiencies and the underlying causative factors were confirmed by direct observation. 


\section{DISCUSSIONS AND CONCLUSIONS}

Evidence suggests that in many healthcare settings existing resources are used less efficiently which could be possible to improve with available resources with re-organization (Trudeau et al., 2010). With growing demands to manage costs and maximize efficiency in acute hospital services, information on patterns of admissions, resource utilization, length of acute hospital stay, need to be carefully studied for better decision making (Moloney et al., 2005). PCU and accident and emergency units in Sri Lanka were conceptualized to function as the casualty unit for all the specialties in a hospital while ensuring efficient service (Sri Lanka Ministry of Health, 2012).

The overburdening emergency department and primary care unit of hospitals represents a universal crisis that negatively influences the quality and access of healthcare (Hoot and Aronsky, 2008). Managing overcrowding in such units, a major barrier to ensure timely and optimum care, needs the proper attention of policymakers (Asplin et al., 2003).

Quantitative measurement of overcrowding was a challenge within the scope of the current study. Possible operational definitions of emergency department overcrowding have been discussed in the world literature according to ambulance diversion, workload measures, length-of-stay of admitted patients, number of clients leaving without being seen, average times before being seen by a healthcare professional, and occupancy rate of stretchers/beds in the emergency department (Schull et al., 2002). Defining overcrowding as a situation where the volume of patients seeking care exceeds the capacity of the health facility, to provide quality care, within an ideal time frame, was a reasonable operational definition of the concept of overcrowding in the emergency department (Drummond, 2002) which was applicable to PCU. In the current study, the overcrowding was assessed on a similar working definition. Having patients waiting to be admitted when all beds were occupied, and the presence of in-patients in 
the PCU without beds were the factors considered to diagnose overcrowding (Makama et al., 2015; Derlet and Richards, 2000; Lynn and Kellermann, 1991).

It was noted that patient flow exceeding the bed capacity led to overcrowding, which was due to waiting for admission, waiting for beds once admitted. Not only the excessive number of admissions but also the waits for discharges and transfers had contributed to overcrowding in the PCU. Increased hospital occupancy is found to be associated with length of stay in emergency departments for admitted patients (Forster, 2003).

Most of the waiting could be at least partly attributed to communication gaps between sending and receiving parties, i.e. doctors in PCU and wards, due to limited direct information sharing, inadequate handoff guidelines and protocols, and limited mutual understanding between clinicians (Abraham et al., 2020).

It was identified in the current study that delays in judicial medical procedures and examination, which must be carried out before discharging the patient, contributed to prolonged stay in PCU. Compared to the western model, a judicial medical officer in state hospitals in Sri Lanka manages both clinical forensic cases as well as post mortems and in most instances burdened with massive workload (Goonerathne, 2011; Edussuriya et al., 2012; Sri Lanka Ministry of Health, 1995). The possibility of arranging an intermediate facility within the hospital to keep the patients waiting to be seen by a judicial medical officer and otherwise fit to be discharged from PCU would ease out crowding.

In a study conducted in the USA it was noted that visits for primary care compared to occurrences of emergencies which are largely random in nature, showed a tendency to accumulate in the day time between the hours of 10 a.m. to 6 p.m., traditionally the peak period of activity. High arrival rates during peak periods in the emergency rooms resulted in a cost to society as each additional patient who joined the queue not only experienced additional waiting 
time but also imposed that burden on all other patients in the unit including those requiring emergency assistance (Siddharthan et al., 1996). The current study did not quantitatively assess the timing of all admissions to conclude the peak hours of arrival of patients, but overcrowding observed in the PCU, DGH Gampaha would have had a similar impact on the overall efficiency of the unit.

It is generally seen in the world literature that compared to younger persons, older adults use emergency services more with longer stays in the emergency department and are more likely to have repeated visits (Aminzadeh and Dalziel, 2002). The age structure of the patients who were admitted to PCU in DGH Gampaha in a randomly selected week in $2018\left(2^{\text {nd }}\right.$ week in the month of October) showed a different picture where most (64.06\%) were 15-64 years old who represented the working population.

The Concept of "Reverse Triage" has been described by researchers to create a classification system for safe early discharge of hospital inpatients to create additional inpatient capacity (Kelen et al., 2006) which could be applied to the current PCU setting as well.

The tremendous benefits of a stat laboratory in or near the emergency department have been discussed repeatedly. Improving the timeliness of laboratory test ordering and turnaround time could be regarded as logical targets for improvement (Yen and Gorelick 2007). Treatment could be begun within minutes when laboratory results are immediately available (Delpachithra and Jayasinghe, 2001) where unnecessary waiting could increase morbidity and, in a compromised patient, mortality as well. Patient disposition would be enhanced by the services of a stat laboratory (Hardin, 1996). The PCU DGH Gampaha did not have a dedicated laboratory within the unit nor the expressed channel for laboratory investigations.

Care coordination failures are attributed to limited interactions among physicians and other health care workers, limited awareness of availability/non-availability of resources, poor 
adherence to patient transfer procedures (Hill, 2005) and monitoring protocols, and limited access to transfer equipment and supplies. These failures have been shown to result in poor care continuity outcomes, inappropriate use of time and hospital resources, increased length of inpatient stay and patient/family anxiety and dissatisfaction. The waits for consultants' opinions, delays in taking over to wards, and delays in judicial medical procedures are facets of the poor coordination issue. Poor teamwork could be detrimental to communication, and coordination (Risser et al., 1999).

The limitations of the current study include lack of patient experience component and inability to ascertain patient contact time during a PCU visit due to retrospective data collection. It was also not possible to objectively quantify the level of overcrowding in the PCU within the scope of the current study.

The optimum service delivery of PCU was challenged by overcrowding and excess waiting. Overcrowding of PCU was mainly due to less organized process flow and inadequate resource availability. Transfer of information, responsibility, and control between sending and receiving clinicians is key to effective patient care between the pre-transfer, transfer, and posttransfer phases among PCU and wards. Strategies to address challenges related to service delivery are the point of care laboratory testing, better communication, standard protocols in management and re-organizing available resources.

Ethical Approval: Ethical approval for the current study was granted by Ethics Review Committee of Medical Research Institute of Sri Lanka (Project number 17/2018) at the meeting held on $11^{\text {th }}$ December 2018.

Authors' Contributions: SMNSM and GSKD took part in the design and conception of the study. SMNSM, CHM and VNN involved in data collection. SMNSM and CHM revised the 
manuscript critically for intellectual content. GSKD did the overall supervision of the study.

All authors read and approved the final manuscript.

Funding and Acknowledgment: The authors acknowledge the administrative support extended by the Medical Superintendent, DGH, Gampaha.

Conflict of Interest Statement: The authors declare that they have no competing interest.

\section{REFERENCES}

Abraham, J., Burton, S., Gordon, H. S. (2020). Moving patients from emergency department to medical intensive care unit: Tracing barriers and root contributors. International Journal of Medical Informatics, 133,104012.

Central Intelligence Agency. (2020, April 6. People and society-Sri Lanka. The World Factbook. https:/www.cia.gov/library/publications/the-world-factbook/geos/ce.html

Delpachithra, C., Jayasinghe, S. (2001). Overcrowding and 'floor" patients in state hospitals: Institutions can make a difference. Ceylon Medical Journal, 46,58-60.

Derlet, R. W., Richards, J. R. (2000). Overcrowding in the nation's emergency departments: Complex causes and disturbing effects. Annals of Emergency Medicine, 35,63-68.

Drummond, A. J. (2002). No room at the inn: Overcrowding in Ontario's emergency departments. CJEM, 4,9197.

Edussuriya, D. H., Marambe, K. N., Abeyasinghe, N., Jayawickramarajah, P. T. (2012). Competencies expected from a medical officer in performing medico legal duties: The results of a Delphi study conducted among judicial medical officers in Sri Lanka. Sri Lanka Journal of Forensic Medicine, Science and Law, 3,6-14.

Forster, A. J. (2003). The effect of hospital occupancy on emergency department length of stay and patient disposition. Academic Emergency Medicine, 10,127-133.

Goonerathne, I. (2011). Challenges towards an optimum in scientific scrutiny of forensic investigations in Sri Lanka. Sri Lanka Journal of Forensic Medicine, Science and Law, 2,11-14.

Hardin, E. (1996). Emergency medicine and the laboratory. Journal of the National Medical Association, 88,279282.

Hill, B. (2005). Optimization of interhospital transfer of patients to reduce emergency department overcrowding. Electronic Theses and Dissertations, 619.

Kelen, G. D., Kraus, C. K., McCarthy, M. L., Bass, E., Hsu, E. B., Li, G., Scheulen, J. J., Shahan, J. B., Brill, J. D., Green, G. B. (2006). Inpatient disposition classification for the creation of hospital surge capacity: A multiphase study. The Lancet, 368,1984-1990.

Lynn, S. G., Kellermann, A. L. (1991). Critical decision making: Managing the emergency department in an overcrowded hospital. Annals of Emergency Medicine, 20,287-292.

Makama, J., Iribhogbe, P., Ameh, E. (2015). Overcrowding of accident and emergency units: Is it a growing concern in Nigeria? African Health Sciences, 15,457. 
Sri Lanka Ministry of Health (2020, April 6). Facilities offered at different categories of Medical Care Institutions. http://www.health.gov.lk/CMS/cmsmoh1/viewcircular.php?cno=01-18/2020\&med=english

Sri Lanka Ministry of Health (1995). Manual of management of Teaching, Provincial, Base and Special Hospitals. Sri Lanka: Colombo.

Sri Lanka Ministry of Health (2012). General Circular No:01-02/2012 Establishment and Management of Preliminary Care Units in Government Hospitals. Sri Lanka: Colombo.

Sri Lanka Ministry of Health (2016). Accident and emergency care policy of Sri Lanka. Sri Lanka: Colombo.

Risser, D. T., Rice, M. M., Salisbury, M. L., Simon, R., Jay, G. D., Berns, S. D. (1999). The potential for improved teamwork to reduce medical errors in the emergency department. Annals of Emergency Medicine, 34,373-383

Schull, M. J., Slaughter, P. M., Redelmeier, D. A. (2002). Urban emergency department overcrowding: Defining the problem and eliminating misconceptions. CJEM, 4,76-83

Trudeau, M., Holmes, M., Bodie, K., Porter, G., Sullivan, V., Tarasuk, J., Trembley, J. (2010). Optimizing resource allocation and patient flow: Process analysis and reorganization in three chemotherapy outpatient clinics. Healthcare Quarterly, 13,48-55

World Health Organization. (2017, April 6). Primary Health Care Systems (PRIMASYS)-Case study from Sri Lanka. https://www.who.int/alliance-hpsr/projects/alliancehpsr_srilankaabridgedprimasys.pdf?ua=1 